\section{Star Formation and Gas Distribution in the Magellanic Spiral NGC 4214}

\section{J. McIntyre}

Physics Department, University of Wollongong, Northfields Ave, Wollongong, NSW 2522, Australia. vjm@davinci.sci.uow.edu.au

Abstract: I present new high-resolution observations of the H I in NGC 4214 made as part of a study of the small-scale kinematics and star formation regions of the galaxy.

\section{Introduction}

A vital part of understanding the evolution of distant galaxies is the detailed study of star formation in nearby galaxies. Simple gravitational instability models are able to explain the large-scale distribution and variation in rate of star formation within the disks of nearby spirals (Kennicutt 1989). However, this model applies only to scales $>1 \mathrm{kpc}$, and there are some notable exceptions to the rule (e.g. M33, Kennicutt 1989), where the total gas surface density is everywhere below the critical value predicted from the rotation curve, but rapid star formation is occuring. Other processes, operating on smaller scales, may also be important in creating new regions of star formation. In particular, sweeping of gas by wind- and supernova-driven supershells has often been proposed as a means of continuing star formation in galaxies (e.g. Tenorio-Tagle \& Bodenheimer 1988). This process may dominate in the extreme late-type galaxies and dwarfs, making them useful laboratories for studying it, relatively undisturbed by large-scale dynamical effects.

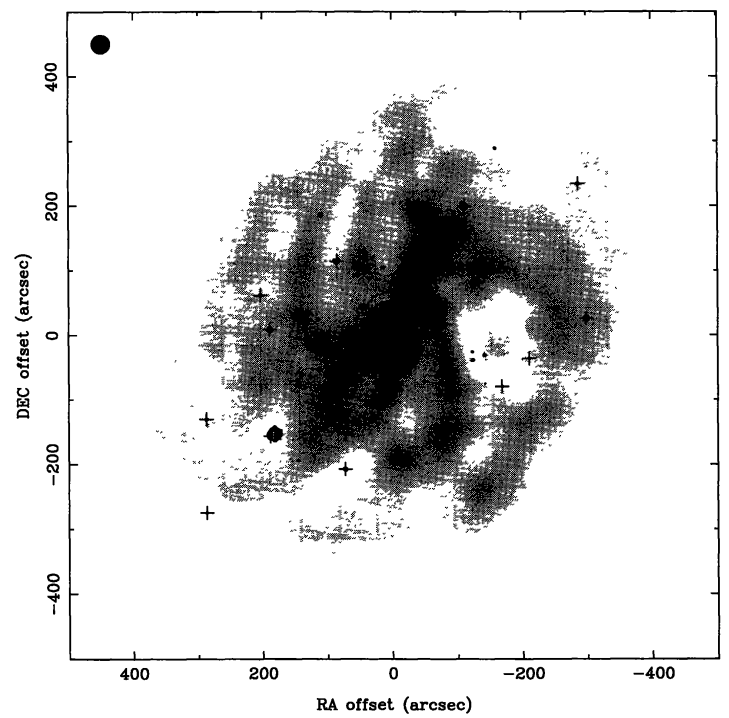

NGC 4214 is a magellanic spiral or irregular in the Canes Venatici I group, at a distance of $\sim 4 \mathrm{Mpc}$. I observed the galaxy with the B, C and D configurations of the Very Large Array (VLA) in 1993 and 1994, in order to study the supershell population and detailed kinematics of the neutral gas. A full account of the reduction and analysis will be presented elsewhere; in this paper I show some initial results on the overall $\mathrm{HI}$ distribution and velocity field.

\section{Results}

The neutral gas column density, compared with the B-band optical emission, is shown in Figure 1. There are several large gaps in the distribution, and a strong impression of multi-arm spiral structure. However, these 'arms' make no impression on the velocity field (Figure 2). Curiously, the sense of curvature in the $\mathrm{HI}$ is opposite to that of the stubby optical 'arms' noted by e.g. Sandage \& Bedke (1995). Despite the appearance of Figure 1, inspection of the channel maps and position-velocity cuts through the cube found that few of the holes in the disk could be clearly identified as supershells; this may be due to a combination of beam smearing and sensitivity limits.

Outside the central $\mathrm{H}_{\mathrm{I}}$ clumps, star formation is concentrated in the $\mathrm{H}_{\mathrm{I}}$ ridge running along the kinematic minor axis $\left(\mathrm{PA} \sim 160^{\circ}\right.$ ) - there is almost none associated with the other 'arms'. Using the rotation curve derived below, I calculated that the critical density for gravitational instability was several times higher than the azimuthally averaged $\mathrm{H}_{\mathrm{I}}$ column density, at all radii. However, in the $\mathrm{H}_{\mathrm{I}}$

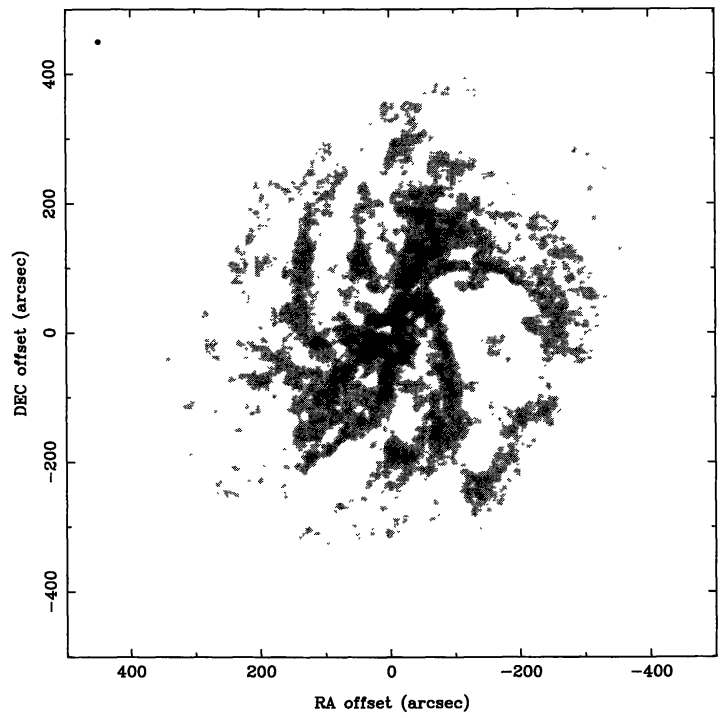

Figure 1-Total H I column density maps of NGC 4214, at $30^{\prime \prime}$ (left) and $8^{\prime \prime}$ (right) resolution. To show maximum detail, different greyscales have been applied to each image. The contours show the outline of the galaxy in the Johnson B band; field stars are marked with small crosses, the rotation centre with a large cross. 


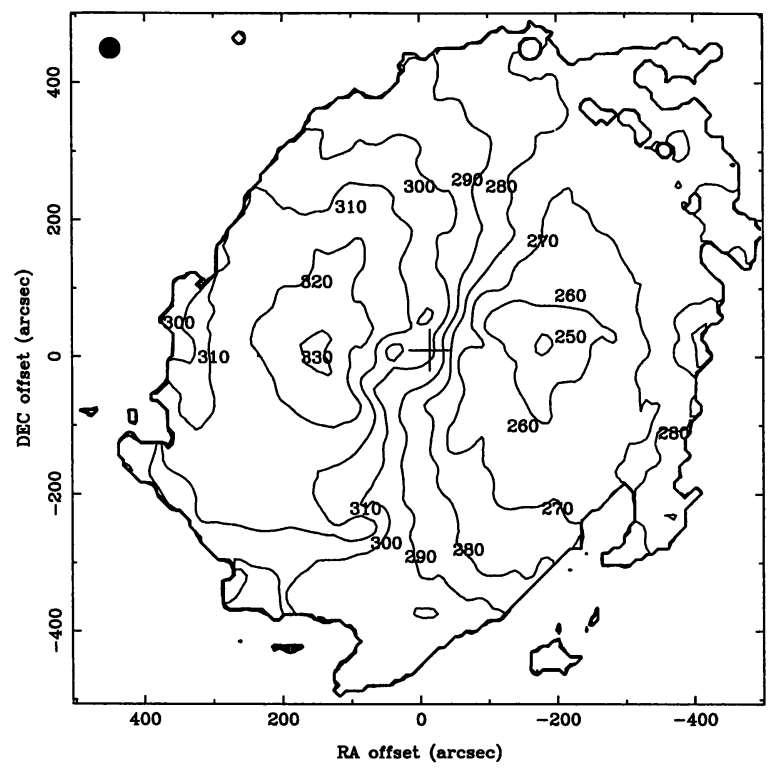

Figure 2-Velocity field of NGC 4214, at $30^{\prime \prime}$ resolution. The cross marks the rotation centre obtained from the model fit described in the text.

ridge, the surface density is within a factor of 2 of the critical value. Considering the uncertainties and lack of data on the distribution of the molecular gas component, it seems possible that the outer star formation regions NGC 4214 can be explained by gravitational instability.

The striking features of the velocity field (Figure 2) are the strong decline in the projected velocity at large radii, and the twist of the isovelocity contours in the central regions. As discussed by Allsopp (1979), there is a number of ways in which such a velocity field could be produced. A warp of the disk to lower inclination at large galactocentric radius seems quite likely, given that deep CCD images (McIntyre 1997, in preparation) show increasingly circular isophotes in the region where the rotation curve is falling. How much of a warp is required depends on the inclination of the galaxy as a whole, which is uncertain. For an inclination of $30^{\circ}$, the inclination of the outer disk must decrease by $15^{\circ}$ over the outer $200^{\prime \prime}$.

However, a warped-disk model cannot explain the strong central isovelocity twist. I have attempted to model the velocity field as a disk with oval orbits in the central regions, that smoothly become more circular with increasing galactocentric radius. The orbit shapes are parametrised in a way similar to that of Staveley-Smith et al. (1990). I took the inclination to be constant at all radii and fitted the shape of the rotation curve with a Brandt (1960) parametrisation. This assumption does not significantly affect the fit to the central regions, as the region of interest is well within the turnover radius. Since it is rather low, the inclination was not fitted automatically; several fixed values were tested. All the free parameters were fitted together, using a simulated annealing algorithm adapted from Press et al. (1992) to minimise the rms difference between the computed and observed velocity field. The best fits were for orbits with rather low ellipticity $(e \leq 0.05-0.10)$ with major axes along $\mathrm{PA} \simeq 110^{\circ}$, roughly parallel to the long axis of the bright optical bar. The orbits were significantly oval only for $R \leq 60^{\prime \prime}$. The fitted rotation curve has a Brandt $n$ parameter of $\sim 10$, and peaks at $\sim 35 / \sin (i) \mathrm{km} \mathrm{s}^{-1}, 160^{\prime \prime}$ from the rotation centre.

\section{Acknowledgments}

I am grateful to NRAO for observing time and to Daniel Puche for teaching me how to use the VLA. This work was made possible by financial support from an Australian Postgraduate Research Award, the Smithsonian Institution Predoctoral Fellowship program and the hospitality of the Australia Telescope National Facility. It has been improved through discussions with Frank Briggs, Lister Staveley-Smith and Wilfred Walsh.

Allsopp, N. 1979, MNRAS, 188, 765

Brandt, J. 1960, ApJ, 293, 131

Glazebrook, K., Ellis, R. S., Santiago, B. X., \& Griffiths, R. E. 1995, MNRAS, 275, L19

Kennicutt, Jr, R. C. 1989, ApJ, 344, 685

Press, W., Teulkolsky, S., Vetterling, W., \& Flannery, B. 1992, Numerical Recipes, 2nd edn (Cambridge University Press)

Sandage, A., \& Bedke, J. 1995, The Carnegie Atlas of Galaxies (Washington: Carnegie Institution)

Skillman, E. 1984, in Star Formation in Galaxies, ed. C. Lonsdale-Persson (NASA-CP 2466), 263

Staveley-Smith, L., Sharples, R., Bland, J., Axon, D., \& Davies, R. 1990, ApJ, 364, 23

Tenorio-Tagle, G., \& Bodenheimer, P. 1988, ARAA, 26, 145

\section{A UKST H $\alpha$ Survey of the Galactic Plane}

\section{Q. A. Parker}

Anglo-Australian Observatory, Coonabarabran, NSW 2357, Australia. qap@aaocbnu1.aao.gov.au

\section{Q. S. Phillipps}

University of Bristol, Tyndal Avenue, Bristol, UK.

Abstract: We describe a major Anglo-Australian project to undertake a UKST H $\alpha$ survey of the Southern Galactic plane, Magellanic clouds and selected regions. The survey will use a new $12 \times 12$ inch monolithic $\mathrm{H} \alpha$ interference filter of high specification and Tech Pan film which offers significant advantages over other emulsions due to its peak sensitivity at $\mathrm{H} \alpha$ and its extremely fine grain, high resolution, exceptional DQE, excellent imaging and low noise. A survey of unprecedented area coverage, depth and resolution should result, superior to any previous optical survey of ionised 